Correction

\title{
Correction: Efficacy of transcatheter arterial chemoembolization for liver metastases arising from pancreatic cancer
}

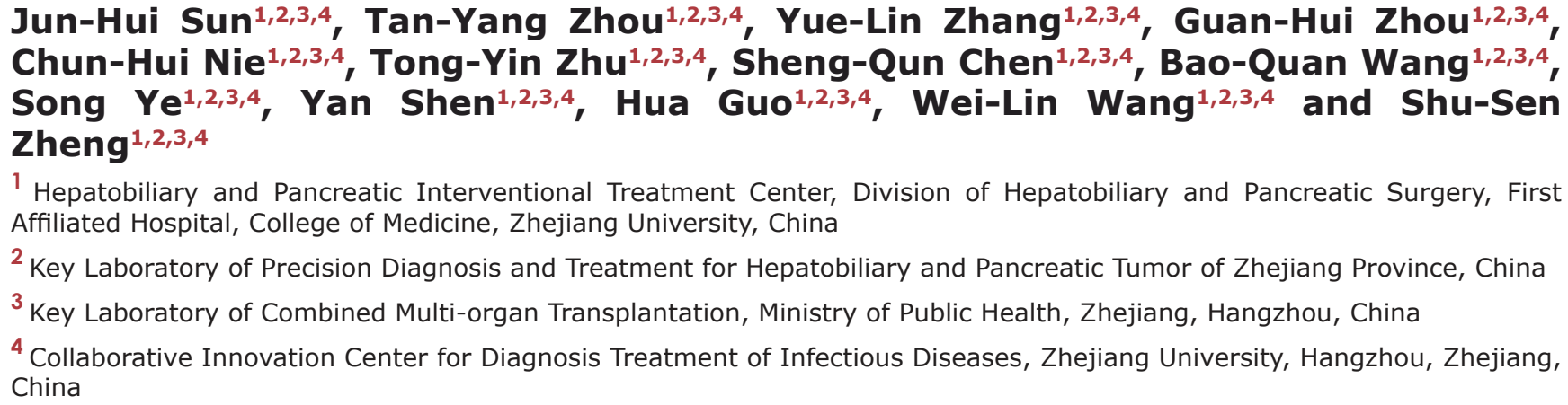

${ }^{1}$ Hepatobiliary and Pancreatic Interventional Treatment Center, Division of Hepatobiliary and Pancreatic Surgery, First Affiliated Hospital, College of Medicine, Zhejiang University, China

${ }^{2}$ Key Laboratory of Precision Diagnosis and Treatment for Hepatobiliary and Pancreatic Tumor of Zhejiang Province, China

${ }^{3}$ Key Laboratory of Combined Multi-organ Transplantation, Ministry of Public Health, Zhejiang, Hangzhou, China

${ }^{4}$ Collaborative Innovation Center for Diagnosis Treatment of Infectious Diseases, Zhejiang University, Hangzhou, Zhejiang, China

Published: March 16, 2018

Copyright: Sun et al. This is an open-access article distributed under the terms of the Creative Commons Attribution License 3.0 (CC BY 3.0), which permits unrestricted use, distribution, and reproduction in any medium, provided the original author and source are credited.

This article has been corrected: The proper name of the institution is as follows:

${ }^{1}$ Hepatobiliary and Pancreatic Interventional Treatment Center, Division of Hepatobiliary and Pancreatic Surgery, First Affiliated Hospital, College of Medicine, Zhejiang University, China

Original article: Oncotarget. 2017; 8:39746-39755. https://doi.org/10.18632/oncotarget.14642 